\title{
A Review on Various Methods used in the Analysis of Bridge Decks
}

\author{
Kishan Gautam \\ Rama University \\ Kanpur, UP
}

\author{
Shashikant Shrivastava \\ Asst. Professor \\ Department of Civil Engineering, \\ Rama University Kanpur, Uttar pradesh
}

\author{
Rohit Rai*, \\ Asst Professor \\ Maharishi University Lucknow UP
}

\begin{abstract}
There are various methods which are used by the researchers for the analyzing of bridge decks. These individual methods have their own advantages and limitations. The methods which have been discussed are Plate Theory, Grillage Method and Finite Element Method. However Nowadays the FE Method is being used commonly due to various reasons, but this paper presents the various work done by the few researches using the mentioned methods and their findings.
\end{abstract}

Keywords-Bending Moment, Torsional Moment, Deflections, Shear Force, Orthotropic Plate.

\section{Literature Review}

Harrop J. (1970) had supplied the strip method of design of skew slabs. The Practical necessities often dictate that during skew slabs the reinforcement be placed parallel to the Sides of the slab in place of in an orthogonal layout, and it's far therefore higher to recall Equilibrium moment fields where the moments are oriented in the reinforcement instructions. The vital equilibrium conditions have been derived and an affinity relationship between skew and square slabs was established. In this situation use become made of the equilibrium equation inside the square coordinate system, because if the slab is to be strengthened with metal parallel to the edges of the slab then it is high quality to apply equilibrium second fields with the moment directions being similar to the reinforcement guidelines. The strip approach offers a relatively simple way of designing strengthened concrete slabs for closing load carrying capacity. The fall apart load of a slab designed by means of this approach will be equal to or more than the design closing load relying on whether or not or no longer the reinforcement is curtailed to give precise correspondence among the equilibrium moment field and the last resistance second area.

The design moments for the radial and angular directions are distributed to the corresponding strips. The cost of material in the curved slab is treated as the objective function that is to be minimized. This will be a function of the design variables that are usually the effective depth and the transformed percentage of steel ratios. The objective function is then minimized subject to the behavior constraints, which are the various limit states, and side constraints. Thus the limit states will define the feasible region in the design variable space within which an acceptable design solution can be found. The search for the optimum is carried out in stages. Initially, the feasible region defined by the critical limit states, namely, the intersection point of the ultimate limit state and one of the serviceability limit states, is considered. The optimum solution for the section at which the maximum moment occurs is obtained in this feasible region. This solution is then checked to see whether it lies in the feasible region defined by the other limit states. In order to determine the optimum solutions in this region, the successive linear programming is introduced as an analytical method of nonlinear optimization. The convergence of the solution is extremely fast;

Four to six iterations were sufficient. The optimization procedure developed in this study can be extended to other curved structural elements. And they also suggested that other design codes may be incorporated by appropriate changes in the program.

Issam E. Harik and Bassam F. Haddad (1986) extended the application of the Analytical Strip Method (ASM) of solution to stiffened sector plates. The plate was idealized as a system of horizontally curved plate strips and beam or rib segments rigidly connected to each other. The behavior of the system was derived by imposing the edge and continuity conditions on the closed form solutions of the individual plate strips and beam elements. The method is applicable to plate-stiffener systems subjected to various loading conditions and with different boundary conditions along the straight and circular edges. The advantage of the ASM solution over the "equivalent" orthotropic plate substitute is that unequally spaced stiffeners and stiffeners of different cross-sectional properties can be introduced in the solution. Results are presented for stiffened sectors with different edge and loading conditions. The ASM can deal with mixed boundary conditions, uniform loads, line and point loads, Similar to the finite strip and spline finite strip methods, the ASM is limited to clamped and or simple supports along the straight edges.

Issam E. Harik and Joseph M. Abou-Khali (1986) studied horizontally curved plates on elastic foundations. Horizontally curved concrete (or rigid) pavements of highways are problems of considerable practical importance, which were related to the solution of sector 
plates on elastic foundations. The purpose of this research was to present an analytical solution for sector plates that are supported along the edges and resting on an elastic foundation. Effect of the subgrade reaction. Results are presented for sector plates with different edge and loading conditions. Different values for the plate. The solution procedure is an extension of the analytical strip method that was previously developed by Harik. The method is applicable to plates with different edge conditions and subjected to various loading conditions thickness (h) and for the modulus of subgrade reaction $\left(\mathrm{f}_{\mathrm{c}}\right)$ is considered.

Issam E. Harik and Hamid R. Molaghasemi(1988) gave an analytical solution to free vibration of sector plates was presented for the free out-of-plane vibration of thin, isotropic annular sector plates with different edge conditions. The classical method of separation of variables was employed. The basic functions in the tangential direction were chosen as the Eigen functions for vibrating beams. These functions were employed in the finite-strip method. However, the advantage of this method lies in the analytical, instead of numerical, representation of the functions in the radial direction. Satisfaction of the governing differential equation of the plate was achieved by the selection of general time-dependent functions and the derivation of appropriate analytical radial functions in terms of Bessel functions. Sector plates of different radii and outer-radius-to-thickness ratios, and with various end conditions along the straight and circular edges had been analyzed. Wherever possible, results are compared to previously published ones.

Mohammad, Khaleel and Rafik (1990) [ (3)] performed finite-element evaluation to decide moments in non-stop right and skew slab-and-girder bridges due to live loads. They analyzed 112 continuous bridges, each having five pretensioned I girders. The spans vary among 24.4 and 36.6 $\mathrm{m}$ and are spaced among 1.8 and $2.7 \mathrm{~m}$ on center. The skew perspective varies between zero and $60^{\circ}$. The finite element analysis uses a skew stiffened plate which consists of thin shell factors and one beam detail. The two skinny shell elements are connected to the beam detail by inflexible links. The element used to model the strengthened concrete deck slab is an eight node isoperimetric skinny shell element with six degrees of freedom at each node. In this study it's miles concluded that large skew angle drastically reduces the layout longitudinal moment. The reduction of maximum tremendous and bad moments within the indoors girders is less than $6 \%$ for skewness of much less than $30^{\circ}$ and as a lot as $29 \%$ when skew attitude is $60^{\circ}$. The discount of the maximum high-quality or poor bending moments in the outdoors girders is much less than $10 \%$ for angles of skew less than $45^{\circ}$ and as a lot as $20 \%$ while skew perspective is $60^{\circ}$.

Alaa Aly and John B. Kennedy (1992) worked on the study of rigidities of horizontally curved flat structures. To predict accurately the response of such curved structures to static and dynamic loads, it is essential to use accurate estimates of the structure's various rigidities. In their study, analytical expressions are derived for predicting the flexural and torsional rigidities during the precracking and postcracking stages. The experimental program consisted of nine tests on one-eighth-scale, horizontally curved, concrete waffle slabs. There were three series of tests, each consisting of three types of specimens--two rectangular in plan for the pure bending tests and the third square in plan for the pure twisting test. Results from the experimental investigation confirm the analytical predictions for the various rigidities during the precracking and post cracking stages. It is also shown that the torsional stiffness's of horizontally curved, fiat structures are much higher than previously estimated.

S. F. Ng and X. Chen (1993) analysis of arbitrary Mindlin plates or Bridge decks by spline finite strip method spline finite strip method for the analysis of arbitrary Mindlin plates is presented. The plate is first mapped into a square domain in the natural coordinate plane, by using the cubic serendipity shape function, and the mapped plate is discretized into a number of strips. The displacements of each strip are described by interpolation functions which are given as products of piecewise polynomials and E3 spline functions. The method, in which three independent reference quantities are used to take into account the effects of transverse deformation, is applied to the analysis of single and multi-span fan-shaped bridge decks. A spline finite strip method to analyze moderately thick plates and bridge decks of arbitrary shapes, according to the Mindlin plate theory, is developed in this thesis. The reduced integration technique is used to eliminate 'shear locking' and the penalty function method is utilized to impose boundary condition at the end of strips and intermediate supports.

Dongzhou Huang, Ton-Lo Wang, and Mohsen Shahawyg (1994) investigated the dynamic behavior of horizontally curved I-girder bridges due to one or two trucks (side by side) moving across rough bridge decks. The bridge is modeled as a planar grillage beam system composed of horizontally curved beam elements and straight beam elements. Warping torsion is taken into consideration in the analysis. The analytical vehicle is simulated as a nonlinear vehicle model with 11 independent degrees of freedom according to the HS20-44 truck design loading contained in the American Association of State Highway and Transportation Officials (AASHTO) specifications. Four different classes of road surface roughness generated from power spectral density function for very good, good. Average, and poor roads are used in the study. The analytical results are very significant and show that the dynamic behavior of curved 1-girder bridges is quite different from that of straight girder bridges. The impact factors of bending and shear for inside girders of curved Igirder bridges are significantly smaller than those for outside girders.

Rajan Sen, Mohsen Issa, Xianghong Sun and Antoine Gergess (1994) studied the finite element modeling of continuous posttensioned voided slab bridges two quarterscale, continuous, longitudinally and transversely posttensioned, two-lane, voided-slab-bridge models-one straight and the other curved in plan-were tested to determine the service-load response under symmetric placement of simulated truck loading according to the American Association of State Highway and Transportation 
Officials. Each model was $2.0 \mathrm{~m}$ (6 ft. 7 in.) wide, with two equal spans of $8.0 \mathrm{~m}(26.27 \mathrm{ft}$.) measured along the centerline. The curved model subtended an arc of $40^{\circ}$ on a radius of curvature of $22.92 \mathrm{~m}$ (75.2 ft.). The voided slab bridge was idealized as an orthotropic plate and its elastic response determined from a two-dimensional finite element analysis using isoperimetric, quadrilateral shell elements. The commercially available computer program

ANSYS was used to conduct the analysis. They compared the predictions from the finite element analysis with test results for deflections, reactions, and stresses. The results show reasonable agreement for the straight model but not for the curved model, where the correlation is poorer. This suggests that the equivalent orthotropic plate parameters proposed in the literature need to be modified for applications involving curved structures.

Sengupta (1991, 1995) analyzed isotropic skewed plates using FEA, with two types of Reissner-Mindlin triangular plate factors proposed. The paper offered numerical results for specific skew angles and support situations to demonstrate the effectiveness of the proposed factors. However, simplest skewed thin plate problems have been included inside the paper..

Mabsout et.Al. (1997) compared 4 finite-detail modeling methods to decide load distribution factors for a one-span, two-lane, certainly supported, composite metallic girder bridge. In the primary method, the concrete slab changed into modeled with quadrilateral shell elements, and the metal girders were idealized as space body individuals. The centroid of each girder coincided with the centroid of the concrete slab. In the second one method, the concrete slab was modeled with quadrilateral shell elements and eccentrically and rigidly related to space body individuals which represented the girders. In the 0.33 method, the concrete slab and metal girder internet have been modeled with quadrilateral shell factors. Girder flanges were modeled as space body factors, and flange-to-deck eccentricity turned into modeled by imposing a inflexible link. In the fourth method, the concrete slab become modeled with isotropic, eight-node brick elements; the steel girder flanges and webs were modeled with quadrilateral shell elements. The 4 finite-detail fashions produced comparable load distribution elements. The outcomes indicated that the concrete slab can be modeled with sufficient accuracy as quadrilateral shell factors and the girders as concentric space body elements..

James M.W. Brown john, Jeffery Lee and Bernard Cheong(1997) studied the dynamic behavior of a $100 \mathrm{~m}$ span curved cable-stayed bridge constructed in Singapore has been studied by full-scale testing and analytical models. Comparison of test results and free vibration analyses show that modeling of the deck end fixity is an important factor, while correct modeling of stay cables and stress-stiffening effects is not so important except for identifying cable modes with weak superstructure interaction which may appear in the measured response. Estimates of stay-cable tension obtained from inducing cable vibrations may be inaccurate unless the anchorage stiffness and mass distribution are well known. Despite its limitations the ambient vibration test technique is most appropriate for a structure of this size.

J. Senthilvasan, D.P. Thambiratnam, G.H. Brameld (2001) also investigated Dynamic response of a curved bridge under moving truck load .Dynamic response of bridges is, however, a complex phenomenon and is less understood in curved bridges. In order to enhance our understanding in this area and to calibrate an analytical bridge-vehicle model, an experimental investigation was carried out on a continuous curved concrete bridge. The twin-cell box girder bridge was tested under the passage of a heavy vehicle at different speeds and strains and the deflections were recorded. The experimental results compare reasonably well with those from an analytical bridge-vehicle interaction model and those predicted by bridge design codes, enabling the calibration of the analytical model. It is evident that dynamic amplification depends both on position on span and location on crosssection. Results also show that there are larger dynamic amplifications in strains than those in deflections.

Eduardo DeSantiago, Jamshid Mohammadi, and Hamadallah M. O. Albaijat (2003) conducted FiniteElement analysis of Horizontally Curved Bridges. A series of horizontally curved bridges were analyzed using simple finite-element models. The analyses included using a typical truckload and also the dead load as the primary forces on bridges. In each analysis, the behavior of bridges was investigated, and the major internal forces developed in members were determined. Specifically, an increase in bending moment and the existence of a torsional moment in cases where the horizontal angle of curvature is large (about 20-30 ${ }^{\circ}$ was observed. The significance of these moments, compared with the maximum bending moment of a comparable straight bridge, was noted. Bridges used in the analysis were assumed to be composed of single spans with about $30.5-\mathrm{m}$ (100-ft) span lengths (the chord length was set at $30.5-\mathrm{m}$ for all bridges), steel girders, and a $203-\mathrm{mm}(8-$ in.) reinforced concrete slab. The finite-element analyses consisted of a three-dimensional idealization using simple beam elements to model top and bottom flanges of the girders and plate bending elements modeling the girder webs and the slabs. The analyses revealed that this simple modeling could effectively be used in analyzing curved bridges. The analyses showed that the bending moment in girders of a curved bridge can be about $23.5 \%$ higher compared with moments in girders of a straight bridge of similar span and design configuration. As a result of the curved geometry of the bridge, a torsional moment is developed in the girders. The magnitude of this moment was found to be about $10.3 \%$ of the maximum bending moment of a comparable straight bridge. The vertical deflection of a curved bridge is about $80 \%$ higher than the deflection of a straight bridge. This is when the angle of curvature is $30^{\circ}$ and a reasonable design for lateral bracing support system is used. However, with the increase in the spacing between lateral supports, the increase in the deflection becomes much higher.

Khaloo and Mirzabozorg(2003) [ (5)] have additionally conducted three-for skew virtually supported bridges of concrete decks with 5 concrete I-segment precast 
prestressed girders the use of ANSYS. Span lengths, skew angles, girder spacing, and arrangements of inner transverse diaphragms have been the simple variables. In all fashions two give up diaphragms and distinctive preparations of internal transverse diaphragms are considered. They analyzed Bridges with 3 span lengths of 25,30 , and $35 \mathrm{~m}$. Girder spacings of 1.8 , 2.four and $2.7 \mathrm{~m}$ and skew angles of $0 \mathrm{o}, 30 \mathrm{o}, 45 \mathrm{o}$, and $60^{\circ}$ are chosen in the skew bridge models. Three unique preparations for internal transverse diaphragms are considered. In the first sample the models do now not include any inner transverse diaphragms. In the second sample inner transverse diaphragms are parallel to the axis of the support, and inside the $1 / 3$ sample internal transverse diaphragms are perpendicular to the longitudinal girders. In their,observe they confirmed that the skew attitude of the deck is the most influential thing on load distribution. The load distribution elements of skew bridges are always much less than the ones of right bridges. The load distribution element of outside girders reduces by way of $24 \%$ for a skew angle of $60^{\circ}$ in comparison with proper bridges. In addition the study confirmed the sensitivity of load distribution elements of inner girders with recognize to skew angle. For decks with a skew angle of $60^{\circ}$ the distribution factors decrease by way of $26.3 \%$ in comparison with proper bridges. However, for decks with a skew angle as much as $30^{\circ}$, this effect is insignificant. In this take a look at for gadget without internal transverse diaphragms, the load distribution elements of outside girder aren't influenced considerably whilst skew perspective increases; however, these factors decrease as much as $18 \%$ in inner girders. When the transverse diaphragms are parallel to the axis of the support, load distribution factors of external girders lower up to $19 \%$ and people of the internal girders are approximately regular whilst skew angle increases. When the inner transverse diaphragms are perpendicular to the girders, distribution factors of both internal

Morley $(1962,1963)$ presented relationships between the rectangular and indirect coordinate systems for load responses in skewed plates. This work became began with a governing equation for isotropic skewed thin plates. The governing equation changed into analytically solved the usage of a trigonometric collection and numerically by the use of the finite distinction method. In deriving the governing equation, the Kirchhoff concept changed into carried out which assumes that directly strains perpendicular to the mid-surface (i.E., the transverse normals) remain straight and ordinary to the mid-surface after deformation. Furthermore, it's far assumed that the mid-floor does not deform. The Kirchhoff concept is widely utilized in plate analysis, but suffers from under-predicting deflections whilst the thickness-to-aspect ratio exceeds $1 / 20$ because it neglects the impact of the transverse shear deformation (e.G., Reddy 2007).

Sabeeh Z.Al-Sarraf(2008) analyze anisotropic plates which have different elastic properties and geometry in different direction by using Grillage method. His analysis model consist of 4-sided beams with flexural rigidity and tortional rigidity and 2-diagonal beams with only flexural rigidity. His aim of analysis was to obtain deformation and deflections of the orthographic plate element of the modelled beam. He used to investigate by using software STAAD Pro. 2006. His analysis shows that modified grillage method gives simpler method and adequate results as compare to the Finite element method or Orthographic plate theory which was solved by using Finite Difference Method for this types of bridge.

Ramesh et al. (2008) presented outcomes for the thick plate trouble of numerous shapes with skew the usage of the FEM and a higher-order Reissner-Mindlin triangular plate detail. It become concluded that this detail can are expecting the pressure distribution higher than the most normally used lower-order plate detail because strain resultants contain higher-order derivatives of the displacements

S.-H. KIM, K.-I. CHO, J.-H. WON and J.-H. KIM (2009) carried out a study on thermal behavior of curved steel box girder bridges considering solar radiation .Solar radiation induces non-uniform temperature distribution in the bridge structure depending on the shape of the structure and shadows cast on it. Especially in the case of curved steel box girder bridges, non-uniform temperature distribution caused by solar radiation may lead to unusual load effects enough to damage the support or even topple the whole curved bridge structure if not designed properly. At present, it is very difficult to design bridges in relation to solar radiation because it is not known exactly how varying temperature distribution affects bridges; at least not specific enough for adoption in design. Standard regulations related to this matter are likewise not complete. In this study, the thermal behavior of curved steel box girder bridges is analyzed while taking the solar radiation effect into consideration. For the analysis, a method of predicting the 3 -dimensional temperature distribution of curved bridges is used. It uses a theoretical solar radiation energy equation together with a commercial FEM program. The behavior of the curved steel box girder bridges is examined using the developed method, while taking into consideration the diverse range of bridge azimuth angles and radii. This study also provided reference data for the thermal design of curved steel box girder bridges under solar radiation, which can be used to develop design guidelines.

D.G. Linzell and J.F. Shura (2009) found out that appreciable warping stresses were generated during girder erection; the classical grillage model predictions were less accurate during girder .Erection while the "modified" model predictions were more accurate during deck placement; and the predicted grillage model deflections were smaller for an exterior-to-interior girder erection procedure than an interior-to-exterior procedure.

Michele Fabio Granata, Piercarlo Margiotta and Marcello Arici (2012) conducted a parametric study of curved incrementally launched bridges and they found out that the structural behavior of incrementally launched bridges in the construction stages depends on different parameters involving deck, nose, supports and guide devices, because static schemes vary continuously with the advance of the deck above the piers. For this reason temporary stresses in the deck, during launching, are rather different from those occurring in service life. Horizontally 
curved launched bridges also present the effects of torsion induced by geometric curvature. A parametric study is presented in order to analyze the influence of design parameters on the construction of these bridges. Analyses were carried out by extending to curved beams a procedure based on the Transfer Matrix Method, already known for straight continuous decks. Effects of curvature, nose-deck ratios of length and load, bending and torsional stiffness ratio were taken into account. The results show that maximum torsion values increase with the decrease in the curvature radius $\mathrm{R}$ and with the decrease in the ratio between bending and torsional stiffness. Moreover, with variation in the nose length ratio, the value $1_{n} / 1=0.60$ with respect to the span length, is confirmed as the optimal value, as happens for straight bridges. With variation in the nose weight, a significant increase in bending moment and torsion can only be appreciated in the cantilever stages of launching. Dimensionless diagrams and related expressions are given for numerical evaluation of the maximum values of bending moment and torsion in the construction stages, with variation in the stiffness ratio and the radius of curvature.

R.Shreedhar and Rashmi Kharde (2013) carried out comparative study of grillage method and finite element method of rcc bridge deck in this they used STAAD PRO and found out that, In general for practical slab bridge deck, result for finite element gives lesser value in terms of bending moment compared with grillage model. Therefore it can be concluded that analysis by using finite element method gives more economical design when compared with the grillage analysis. But the benefit for grillage analysis is that it is easy to use and comprehend.

\section{CONCLUSION}

As in today's scenario we have witness the tremendous increase in the use of computer programs. The old method of plate theory has been completely replaced by modern methods of FE method and Grillage Method and amount two the FE method is more reliable.

But most of designers can go for different computer programs which are available in the market and compare those results for the verification.And as fer as researchers are concern they can go for study of convergence of the results in elastic as well as in inelastic modes in all the three methods.

\section{REFFERENCES:}

[1] HARROP J., (1970) "Ultimate Load Design of Skew Slabs by the Strip Method" Building Science Vol. 5, pp. 117-121

[2] Issam E. Harik (1983) "General Solution to Bending of Orthotropic Sectors" Journal of Engineering mechanics/ Volume 110.

[3] Issam E. Harik and Sasan Pashanasangi (1984) " Curved Bridge Decks: Analytical Strip Solution" Journal of Engineering mechanics/ Volume 111-july 1985

[4] Moon Ho Park and Issam E. Harik (1986) "Optimum Design of Horizontally Curved R/C Slabs" Journal of structural Engineering / Volume 113-nov 1987.

[5] Alaa Aly and John B. Kennedy (1992) "Rigidities of Horizontally Curved Flat Structures" Journal of structural Engineering / Volume 119-Sept 1993

[6] S. F. Ng and X. Chen (1993)" Analysis of arbitrary Mindlin plates or bridge decks by spline finite strip method" Computer and structures Volume 54, Jan 1995.
[7] Dongzhou Huang, Ton-Lo Wang, and Mohsen Shahawyg (1994) "Dynamic behavior of horizontally curved I-girder bridges" Computer and structures Volume 57, Nov 1995.

[8] Rajan Sen, Mohsen Issa, Xianghong Sun and Antoine Gergess (1994) "Finite Element Modeling of Continuous Posttensioned Voided Slab Bridges" Journal of structural Engineering / Volume 120-Feb 1994.

[9] James M.W. Brown john, Jeffery Lee and Bernard Cheong(1997) "Dynamic performance of a curved cable-stayed bridge" University of Exeter-Nov-1999.

[10] J. Senthilvasan, D.P. Thambiratnam, G.H. Brameld (2001) "Dynamic response of a curved bridge under moving truck load" Engineering structures 24 (10), 1283-1293.

[11] S.-H. KIM, K.-I. CHO, J.-H. WON and J.-H. KIM (2009) "A study on thermal behaviour of curved steel box girder bridges considering solar radiation" Archives of Civil and Mechanical Engineering Volume 9, Issue 3, 2009

[12] D.G. Linzell and J.F. Shura (2009) "Erection behavior and grillage model accuracy for a large radius curved bridge" Journal of Constructional Steel Research 66 (2010) 342-350

[13] Khaleel, M.A., and Itani, R.Y. (1990). "Live-Load Moments for Continuous Skew Bridges." Journal of Structural Engineering, 116(9), pp 2361-2373.

[14] Menassa, Mabsout, Tarhini and Frederick.(2006) "Influence of Skew Angle on Reinforced Concrete Slab Bridge" the Journal of Bridge Engineering, March 1, 2007, Vols. Vol. 12, No. 2

[15] Load Distribution Factors in Simply Supported Skew bridges. Mirzabozorg, Khaloo and. s.l. : JOURNAL OF BRIDGE ENGINEERING @ ASCE, July/August 2003, Vols. 8, No-4

[16] Sengupta, D., "Performance study of a simple finite element in the analysis of skew rhombic plates", Computers and Structures, 54(6), pp.1173-1182, 1995

[17] Dr. Sabeeh Z.al-Sarraf(2008) "Analysis of composite Bridge superstructure using Modified Grillage Method", Eng. \& Tech Journal, Vol.27,No.5,2009

[18] Ramesh, S.S., Wang, C.M., Reddy, J.N. and Ang, K.K., " Computation of stress resultants in plate bending problems using higher-order triangular elements", Engineering Structures, 30(10), pp.2687-2706, 2008

[19] Morley, L.S.D., "Bending of a simply supported rhombic plate under uniform normal loading", The Quarterly Journal of Mechanics and Applied Mathematics, 15(4), pp.413-426, 1962

[20] R.Shreedhar,RashmiKharde,2013'Comparative study of Grillage method and Finite Element Method of RCC Bridge Deck' International Journal of Scientific and Engineering Research Feb- 2013 